\title{
Isoniazid, The Frontline of Resistance in Mycobacterium tuberculosis
}

\author{
James B. Whitney B.Sc. ${ }^{\dagger}$ and Mark A. Wainberg Ph.D. ${ }^{* \dagger}$
}

\begin{abstract}
Tuberculosis is an ancient disease that has held close association with humans for millennia. Through persistence, this remarkably successful organism has managed to infect an estimated third of the world's population. Declining rates of tuberculosis in developed nations have masked an emerging epidemic of drug resistant cases that have been reported in almost every country under scrutiny. The recent completion of the genome sequence of Mycobacterium tuberculosis has mandated more efficient control and management of this disease. The momentum for this public health imperative will come from information gleaned from advances in genomics and related technologies towards deciphering molecular mechanisms of mycobacterial drug resistance.
\end{abstract}

\section{INTRODUCTION}

Tuberculosis, a disease of great antiquity holds lineage to saprophytic soil organisms whose later introduction as a human pathogen likely coincided with the domestication of cattle approximately 10,000 years ago. Throughout history, tuberculosis has been classified by many names, from Phthisis ("to waste") by the ancient Greeks, to consumption in the 1800's. In 1882 Robert Koch isolated the causative agent, Mycobacterium tuberculosis from crushed lung tubercles. Currently, M. tuberculosis is known as the world's leading cause of death from a single infectious agent, with a global prevalence of greater than 1.6 billion persons. $(1,2)$

\footnotetext{
*To whom correspondence should be addressed: Mark A. Wainberg, McGill University AIDS Centre, Lady Davis Institute-Jewish General Hospital, 3755 Cote Ste-Catherine Road, Montreal, Québec, Canada, H3T 1E2. Telephone: (514) 340-8260. Fax: (514) 340-7537. E-mail: mark.wainberg@mcgill.ca.

$†$ McGill AIDS Centre, Lady Davis Institute-Jewish General Hospital and Department of Microbiology and Immunology, McGill University, Montreal, Quebec Canada H3A 2B4.
}

Despite overall declines in $\mathrm{TB}$ incidence in industrialized countries during the past three decades, mostly due to conscientious public health measures, there has recently been an important rise in the incidence of TB. $(3,4)$ However, the contention that this is a "new" epidemic may not be entirely accurate when one considers the natural history of this disease follows secular trends that epicycle over the course of a human lifespan. What is "new" about this increasing disease burden is the widespread emergence of multi-drug resistant strains (MDR), which by definition, are strains resistant to at least the major frontline drugs: isoniazid and rifampin. This increase is due, at least in part, to the discontinuation of long course multi-drug treatments combined with patient non-compliance (5). In addition, the predilection of M. tuberculosis for the impoverished has further compounded this problem (6). As well, $M$. tuberculosis exhibits an important synergy with HIV and the role of the latter as a cofactor in TB disease has proven to be a major impediment to the control of both AIDS and Tuberculosis. $(4,7,8)$

Nonetheless, it is apparent there has been an increased incidence of MDR TB in both developing and 

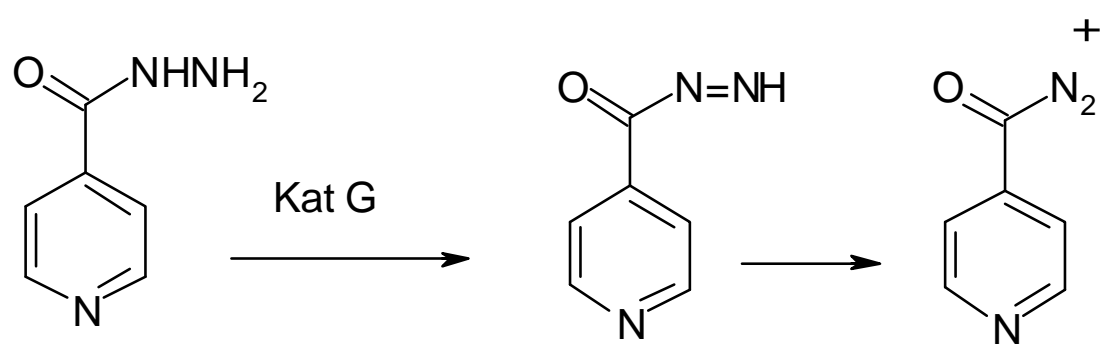

Figure 1. Potential metabolic activation mechanism for Isoniazid. Kat $G$ mediates 2 electron transfers to produce an activated Isoniazid intermediate(s). It is this reactive intermediate that is capable of intracellular acylation of nucleophiles in M.tuberculosis, thereby facilitating toxic effects.

industrialized countries, despite the availability of directly observed therapy (DOTS) and BCG vaccine. These recent developments underscore the urgent need not only for new drugs and more efficacious vaccines, but more importantly, a concerted effort into devising methods for timely vaccine and chemotherapeutic development. In light of this urgent need, it may be serendipitous that we are seeing enormous leaps in proteomic and genomic technologies as well as the complete published sequences of the M. tuberculosis (H37RV) genome $(9,10)$.

Therefore, this review is intended to consider previous work in light of recent advances using the major frontline antitubercular drug, isoniazid, as a paradigm for mycobacterial drug resistance. Following an abridged historical development of isoniazid, the current understanding of its mechanism of action and mechanism(s) of resistance and the rationale for the exquisite sensitivity of $M$. tuberculosis to isoniazid will be discussed. From there, the current directions of TB research in conjunction with present genomic based approaches will be considered.

\section{ISONIAZID AND THE ROLE OF KatG}

Isoniazid (INH), or isonicotinic acid hydrazide (Figure 1), is a synthetic bactericidal agent that was first produced in the early 1900's but was not utilized as an antitubercular agent until 1952. Presently, it is the prophylaxis of choice due to its low cost per dose, relatively low frequency of hepatotoxicity, $(11,12)$ and reasonable bioavailability (13). In conjunction with Rifampin and Pyrazinamide it forms the major front line therapy worldwide (14).

INH enters mycobacterial cells via passive diffusion across the bacterial envelope (15). The minimal inhibitory concentration (MIC) for susceptible strains ranges from $0.02-0.05 \mathrm{mg} / \mathrm{ml}$ and is equally effective in M. tuberculosis and M. tuberculosis complex (M. bovis, M. microti, M. africanum) members. Surprisingly, INH exhibits little or no inhibitory activity against other mycobacteria and most prokaryotic pathogens. The reasons for this, and the primary mechanism of action of
INH have been the subject of considerable investigation. Much of the current understanding of the in vivo mechanism of isoniazid has been extrapolated from in vitro work; almost exclusively focusing on relatively few bacterial enzymes associated with decreased susceptibility profiles. The first mechanistic insight of INH was revealed in 1954 when Middlebrook and others noted an inverse relationship to catalaseperoxidase activity and INH resistance $(16,17)$.

This implicated the catalase-peroxidase enzyme, or KatG of M. tuberculosis. KatG is a hemeB containing dimer with only one functional domain. The other is apparently inactive (18). Its physiological role is protective, combating the low $\mathrm{pH}$ found during the "oxidative burst" in human phagocytes, where liberated $\mathrm{O}_{2}$ radicals are converted to $\mathrm{H}_{2} \mathrm{O}_{2}$ within the phagosome. KatG activity eliminates this via a "deceptively simple reaction" (18);

$$
2 \mathrm{H}_{2} \mathrm{O}_{2} \rightarrow 2 \mathrm{H}_{2} \mathrm{O}+\mathrm{O}_{2}
$$

Which is in fact a 2-step prosthetic group mediated process:

$$
\begin{aligned}
& \text { Heme-Fe[III] }+\mathrm{H}_{2} \mathrm{O}_{2} \rightarrow \text { Heme-Fe[IV] }+\mathrm{H}_{2} \mathrm{O} \\
& \text { Heme-Fe[IV] } \left.+\mathrm{H}_{2} \mathrm{O}_{2} \rightarrow \text { Heme-Fe[III }\right]+\mathrm{H}_{2} \mathrm{O}+\mathrm{O}_{2}
\end{aligned}
$$

It is clear that KatG plays a pivotal role in virulence and has been found to be essential for persistence in mouse and guinea pig models $(18,19)$.

Curiously, it is this same protective enzyme that is implicated in susceptibility to INH. Specifically, INH is a prodrug that requires cellular activation by $\mathrm{KatG}$ producing a reactive species with antimicrobial action. The postulated reaction is shown in Figure 1. There is also evidence that INH effect is potentiated by the presence of peroxide, typically found in activated macrophages $(20,21)$.

An elegant series of experiments by Zhang et al. demonstrated a key role for KatG in the action and resistance to INH. This group sought to identify the gene(s) responsible for INH resistance. A molecular 
genetic approach was taken using a lab adapted $M$. smegmatis strain normally susceptible to moderate concentrations of INH $(32 \mathrm{mg} / \mathrm{ml})$. From this, a mutant strain was isolated that was viable in concentrations up to $500 \mathrm{mg} / \mathrm{ml} \mathrm{INH}$, this particular mutant was transformed with a cosmid library representing the entire genome of the INHs lab strain H37RV. Selection with INH allowed isolation of a hypersensitive clone, that displayed a marked catalase activity, thereby co-locating these two functions to a single genomic fragment (22).

Restriction mapping and Southern blotting localized the INH susceptibility to a smaller $2.9 \mathrm{~Kb}$ fragment, which in comparison with $E$. coli was shown to contain sufficient coding capacity for KatG. In addition, Zhang et al. provided evidence that over expression of this product could confer a susceptible phenotype in a dose dependent manner to a naturally $\mathrm{INH}^{\mathrm{R}} E$. coli strain.

To determine the clinical relevance of this result, a series of eight $\mathrm{INH}^{\mathrm{R}}$ clinical isolates were assayed by southern analysis confirming that high resistance of two strains was due to deletion of the KatG gene. It is worth noting at this point that gene deletion is a relatively unusual mechanism of resistance in contrast to what is typically observed in bacterial systems where active efflux, (23) altered affinities of enzymatic targets, (24) and antibiotic inactivation, (25) represent norms in resistance acquisition.

Therefore, although convincing, it is likely that the studies conducted by Zhang et al. suffered from errors in sampling since this mechanism of resistance appears in a distinct minority of cases.

A more representative sample was analysed by Heym et al. (26). This group used 39 clinical isolates from diverse locales to determine whether mutations in KatG were associated with $\mathrm{INH}^{\mathrm{R}}$. A PCR-SSCP (PCR-single stranded conformational polymorphism) strategy was used, in which the target is first amplified by PCR. Products are then denatured to single -stranded form and run on high-resolution polyacrylamide gels. Sequence alterations can be inferred by altered mobilities in comparison to reference standards. Mutations are then typically confirmed by sequencing (For detailed reviews see 27, 28).

This methodology allowed rapid screening of resistant and control isolates in 12 arbitrary overlapping intervals. Aberrant mobilities were sequenced and indicated that 21 of the 36 resistant isolates contained mutations within KatG with an Arginine to Leucine mutation at position 463 predominating in seven isolates. Five others carried a Serine to Threonine mutation at position 315. An additional three isolates contained a deletion mutation at residues $120-123$. The final six mutations in KatG were represented at a singular frequency.

Similar, but less convincing work was reported by Pretorius et al., using a lower resolution PCR-SSCP
Table 1. Relative activities and isoniazid minimal inhibitory concentrations (MICs) of Bacillus Calmette Guerrin BCG transformants expressing KatG mutants versus reference lab strains.

\begin{tabular}{llll}
\hline Strain & Peroxidase (A405) & Catalase (A240) & $\begin{array}{l}\text { Isoniazid MIC } \\
\text { (mg ml -1) }\end{array}$ \\
\hline VC & & & $>500$ \\
TB-KatG & 0.0 & 0.0 & 0.5 \\
MAC-KatG & 1.0 & 1.0 & 1.0 \\
R104L & 1.9 & 1.2 & $>500$ \\
H108Q & 0.1 & 0.2 & $>500$ \\
N138S & 0.1 & 0.0 & $>500$ \\
L148R & 0.1 & 0.0 & $>500$ \\
H270Q & 0.1 & 0.2 & $>500$ \\
T275P & 0.1 & 0.0 & $>500$ \\
W321G & 0.3 & 0.0 & $>500$ \\
D381G & 0.3 & 0.3 & $>500$ \\
S315T & 0.0 & 0.0 & 90 \\
S140N & 0.6 & 0.4 & 0.5 \\
A350T & 1.6 & 1.5 & 1.0 \\
R463L & 1.3 & 1.0 & 0.5 \\
R463G & 1.9 & 1.4 & 0.5 \\
L587M & 0.9 & 1.3 & \\
\hline
\end{tabular}

Adapted from Rouse et al. (1996). Of note is the S315T mutation that, while conferring only moderate resistance to isoniazid ( $\sim 90 \mathrm{mg} \mathrm{ml}-1)$, allows retention of significant levels of associated peroxidase and catalase activities. MIC, minimal inhibitory concentration.

methodology $(29,28)$. However, the strength of this work was in the relatively large and geographically diverse samples from Africa, the US and Switzerland. Results of this study and a later one show a high proportion $(52 \%)$ of African isolates with Serine 315 Threonine (G-C) mutations at codon 315, and additional mutations at codons Thr 275 Ala, Arg 409 Ala, Arg 463 Leu and Asp 695 Ala. Overall, 64\% of the observed INH resistance was attributable to mutations within KatG, again suggesting a complete KatG deletion was a rare event. An interesting additional finding of this report was that of a fully INH susceptible control isolate mutated at codon 463, which will be discussed later.

Numerous recent investigations have led to similar conclusions. Martila et al. found 22 of $24(91.7 \%)$ INH resistant isolates carried a Ser 315 Thr mutation, of which 12 also carried a 463 mutation, all originating from the St. Petersburg area in Russia (30). Clonal spread in this case was not markedly noted by the authors in this study. However, due to the genetic similarity of isolates, it would have been interesting if some evidence had been presented regarding the transmission of drug resistant strains. Unfortunately, studies of clonal transmission of resistant bacteria in this area were not investigated.

The mutational spectrum of KatG may also be a factor of geography as the aforementioned author several years previous found a low prevalence, (three of fifty-four isolates containing mutations at codon 315) in Finnish patients (31). A similar result was also obtained by Rouse et al. in a clinical study of $26 \mathrm{INH}^{\mathrm{R}}$ isolates, nineteen of which were from Korea with only a single isolate mutated at codon 315 (33). However, it appears the study may have been limited by the use of isolates exhibiting rather low inhibitory concentrations of $\sim 1 \mathrm{mg} / \mathrm{ml}$. In contrast, an opposite 
<smiles>NNC(=O)c1ccncc1</smiles><smiles>CCc1cc(C(N)=S)ccn1</smiles>

Figure 2. Structures of Isoniazid (INH) and Ethionamide (ETH/ETA)

finding was noted in studies in the Netherlands that showed distinctly higher levels of clinical isolates carrying 315 mutations (32).

Overwhelmingly, the predominance of the 315 mutations in clinical INH $r$ is supported in several other large and well-conducted studies. Haas et al. (34) which concluded $64 \%$ of KatG mediated resistance was due to mutation of codon 315 , as well as an older but extremely convincing study by Musser et al. (35) indicating $75 \%$ of $\mathrm{INH}^{\mathrm{R}}$ isolates contained the 315 or 463 codon mutations.

In considering previous studies, several investigators noted that the majority of mutations seen clinically were of the missense type. This indicated the importance of maintaining some, albeit reduced, KatG function in vivo. The rationale behind this is that even low KatG activity still confers a selective advantage versus nonsense mutations that result in truncated products.

Rouse et al. validated this hypothesis in a welldesigned study that investigated the impact of specific missense mutations on KatG function. This was accomplished by introducing fourteen genetically defined mutations at thirteen different codons (36). The relative effects of both in vitro enzyme assays and ex vivo INH resistance were compared as shown in Table 1. This data reveals the benefit of the Ser 315Thr mutation in contrast to other mutations, which abrogate catalase activity or result in relatively insignificant increases in MIC's. However, this last point may be confounded by the use of the BCG as a transformation target. BCG in general is comprised of a phylogenetically distinct group of attenuated species, harboring a series of mostly uncharacterized deletions $(37,38)$. This may have some effect on the validity of studies. However, relative concordance between data using this and other laboratory adapted species is reassuring.

Overall, Rouse's data is in agreement with recently published data by Wengenach et al. (39). Her report included a thorough biochemical analysis of the properties of the Ser 315Thr mutation in comparison to wild type. The results show a six folds drop in catalase activity but only a two folds reduction in peroxidase activity. Taken together, these studies add credence to the hypotheses that the Ser315Thr KatG mutant is a competent catalase peroxidase harboring reduced affinity for INH.
One of the most commonly seen mutations associated with INH resistance in a clinical setting is the Arg 463 Leu or Arg 463 Ala. However, the prevailing opinion is that this mutation does not appear to confer any selective advantage $(39,40)$. In particular, Johnsson et $a l$. found no difference between purified wild type and the R463L KatG mutant in terms of activity or ability to discriminate isoniazid substrate (40). Despite being completely in vitro, this study combined with others $(26,32,33,34,41)$, concludes the most plausible explanation is that the 463 mutation is a frequent and possibly geographically isolated polymorphism. However, it should be stated there might be a marginal decrease in INH susceptibility between the KatG 463 leu KatG 463 glu, since the 463 leu naturally occurs in M. Bovis, which does show slightly higher MIC. However, evidence of this sort is weak (36).

In conclusion, considerable evidence has shown that INH acts as a prodrug that requires activation by KatG. Approximately $60-70 \%$ of all observed INH resistance can be directly linked to defects in KatG. As mentioned, this indicates the likelihood of additional resistance effectors, and raises an important question with the exact target(s) of the activated INH product.

\section{InhA AND FATTY ACID SYNTHESIS}

As stated, mutations in KatG account for approximately two thirds of INH resistance. Therefore, several groups have postulated additional downstream targets of activated INH in mediating resistance $(33,35$, 44). This was initially based on phenotypic evidence that INH affects cell wall synthesis. Also, at a low frequency of bacterial isolations (10-7), INH resistance was not correlated with loss of catalase activity but rather with co-acquisition of ethionamide (ETH) resistance. ETH is a structural analog to $\mathrm{INH}$, indicating a possible common target (Figure 2).

In an effort to identify this target, Banerjee et al. utilized a lab derived spontaneous INH/ETHr mutant to construct a genetic library for complementation studies (42). Two ORFs (open reading frames) were identified, termed orf1 and inhA. Subsequent subcloning studies, using both genomic fragments from $M$. bovis and $M$. tuberculosis $\mathrm{H} 37 \mathrm{Rv}$ cloned into the $M$. smegmatis MC2155 strain revealed several interesting results. The first is that the InhA product alone was sufficient to cause an INH resistant phenotype. Second, in comparison to the $M$. smegmatis gene the, M. bovis and $M$. tuberculosis InhA genes appear to be located within an operon that includes Orf1. Also, the intergenic region for both $M$. bovis and $M$. tuberculosis H37Rv was considerably shorter than that of $M$. smegmatis and may lack complete promoter sequences.

Sequence alignment of InhA showed marked conservation across all mycobacterial strains and 
significant homology (40\% identity) to the E. coli ENV $\mathrm{M}$ protein known to be involved in fatty acid biosynthesis. Sequence comparisons also revealed a single nucleotide change at position 94 from serine to alanine as well as a putative NAD+/NADH binding site. The basis of INH resistance was determined by cell free mycolic acid synthesis assays. In the presence of INH wild type InhA was inhibited in a rough dose dependent manner. In contrast, the S94A mutant strain showed 20 folds greater activity under similar conditions. These data are quite consistent with the supposition that KatG activated INH targets mycolic acid biosynthesis. This group also proposed a hypothetical mechanism based on E. coli ENV M resistance to diazaborine, (42) that was discarded, based on the crystallization and functional analysis of InhA by Dessen et al. (43).

This group confirmed several unresolved questions. First, was the confirmation of the InhA function in mycolic acid biosynthesis. The role of this enzyme was in the catalysis of the NADH specific reduction of 2trans-enoyl acyl carrier protein (ACP), an essential step in fatty acid elongation. Second, it was shown by microcalorimetry that neither unmodified isoniazid nor ethionamide bound to InhA supporting a role for activation of these prodrugs by KatG. The salient feature of this paper was that while the $\mathrm{K}_{\mathrm{m}}$ and $\mathrm{V}_{\max }$ values of the enoyl substrate for the wild type and S94A mutant didn't differ significantly, the $\mathrm{K}_{\mathrm{m}}$ for $\mathrm{NADH}$ was 5 fold higher in the S94A mutant.

This indicated the resistance mechanism was related to specific interplay between the enzyme and cofactor, not INH. Crystallization data from the WT and S94A mutant indicated that perturbations in hydrogen-bonding within the NADH binding site impaired its affinity for NADH. As tantalizing as these results were the complete picture was left to a later report by the same group where an attractive mechanism was put forth (44).

In the WT condition there is a preference for NADH to bind first to InhA, followed by an acyl-ACP substrate, initially leaving the InhA-NADH complex available for attack by activated isoniazid. Due to the higher affinity, the WT InhA-NADH-INH complex would result in permanent inhibition of mycolic acid synthesis. Conversely, the decreased affinity of the S94A mutant would promote acyl-ACP substrates to bind first before NADH thereby protecting the enzyme. Furthermore, in a NADH-INH bound condition the lower affinity may promote release of the inhibitory complex.

Although of enormous academic interest, the clinical relevance of the S94A mutant is questionable since the clinically observed mutations in inhA do not include S94A (44). Rather in this study they are reported at residues $16,21,78$ and 95 . The fact that these are still physically near the NADH binding domain is encouraging (Figure 4). As added proof to the proposed mechanism, a paper by Lei et al. on isolation of the
Table 2. Resistance-associated mutations and amino acid substitutions in the Kas A codon. Adapted from Mdluli et al., 1998. Depicted diagrammatically in Figure 3.

\begin{tabular}{lll}
\hline KasA Codon & Nucleotide D & Amino Acid D \\
\hline 66 & GAT - AAT & D -- N \\
269 & GGT -- AGT & G -- S \\
312 & GGC -- AGC & G -- S \\
413 & TTC -- TTA & F -- L \\
\hline
\end{tabular}

InhA inhibitor complex showed complete inhibition of InhA by the presence of a tightly bound $\left(\mathrm{K}_{\mathrm{D}}<0.4 \mathrm{~nm}\right)$ INH product that had been activated by KatG (45).

\section{THE ROLE OF B-KETOACYL ACP SYNTHASE (KASA) AND INH}

There seems to be considerable dispute within the literature as to additional targets of INH (46). Mudluli et al. has investigated accumulations of a saturated hexacosanoic acid (C26:0) found under INH treatment (47). This saturated fatty acid was found linked to acyl carrier protein (AcpM) that normally runs at $12 \mathrm{KD}$ in SDS-page gels, however a second $80 \mathrm{KD}$ complex with the same amino terminus was found to be a covalent complex of B-ketoacyl ACP (KasA) INH and ACPM. Automated sequence analysis of four $\mathrm{INH}^{\mathrm{R}}$ clinical samples revealed four different mutations at the kasA loci (Table 2 and Figure 3). Notably, two samples with the mutations G269S and F413L carried no other mutations, adding strength to the association. The remaining two strains carried additional KatG 315 mutations.

By comparison to the crystal structure of the E. coli homolog, three of the amino changes were within the catalytic center, the fourth was found to be located at the carboxy terminus and purported to alter protein-protein interactions (47). The clinical importance of KasA in INH resistance still needs to be fully established. A study by Lee et al. may assist in this regard (48). A series of $160 \mathrm{INH}$ resistant isolates were sequenced and $10 \%$ carried mutations in KasA involving R121K, G312S, G387D, and previously reported G269S. However, 6 out of 32 susceptible strains also contained the G312S alteration, a seemingly similar situation to the KatG 463 mutation. Nevertheless, the possibility of KasA constituting a resistance mechanism should not be ruled out completely, since the R121K and G387D mutations have yet to be reported in a susceptible strain, and mutations in KatG and InhA do not account for all $\mathrm{INH}^{\mathrm{R}}$ seen clinically.

Therefore, it seems possible that geographically distinct polymorphisms between epidemiologically disparate strains may be commonplace. One should also consider that both INH action, as well as resistance is likely pleotrophic in nature. This line of thought is supported by a recent study that utilized specific inhibitors of Inh A and KasA to demonstrate that INH 


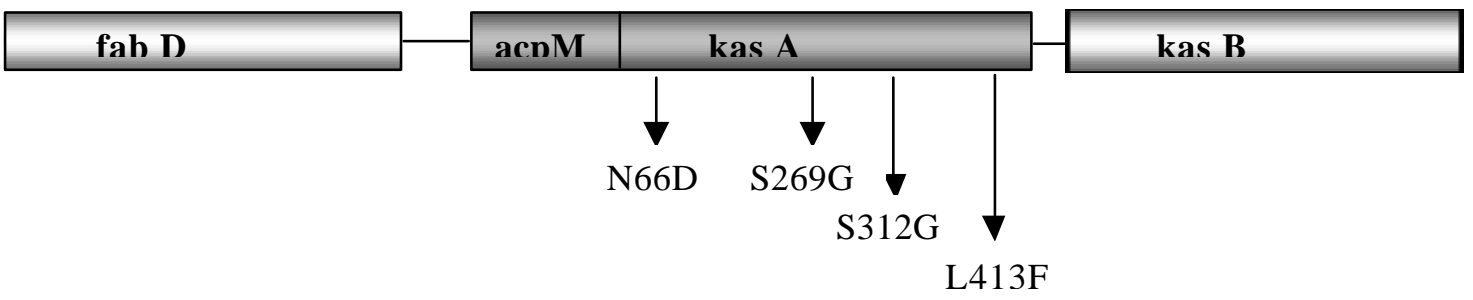

Figure 3. Diagrammatic representation of the kasA codon. Adapted from S. Ramasaswamy et al.,1998.

affects both targets simultaneously albeit by independent mechanisms, thereby arguing convincingly for a role of both targets in INH resistance (49).

\section{THE OXIDATIVE STRESS PARADOX: THE OxyR-AhpC REGULON}

The study of both KatG and InhA has provided valuable clues to the action of INH but has also introduced some unresolved questions. The exceptional sensitivity of $M$. tuberculosis to INH lacks rationale in comparison with other bacteria (54). To persist and flourish pathogenic mycobacteria must be able to withstand high oxidative stresses found in macrophages. In gram-negative organisms such as E. coli and $\mathrm{S}$. typhimurium, the oxidative stress response is controlled by the OxyR protein. OxyR can upregulate several genes including AhpC (encoding acyl hydroperoxidase reductase), Dps (19KD starvation protein), OxyS (a divergent product of oxyR), GorA (glutathione reductase), and most importantly KatG (53). Sherman et al. investigated this response in $M$. tuberculosis and several other mycobacterial members (50). Metabolic labeling experiments were conducted under peroxide challenge and 2D protein gels indicated an OxyR like protective response was upregulated by only $M$. smegmatis; all M. tuberculosis and M. tuberculosis complex members elicited only an upregulated KatG. Deretic et al. and others have investigated the oxidative stress regulon in $M$. tuberculosis to determine the basis for these altered expression patterns $(51,52,54)$. They specifically sequenced the AhpC gene and its putative regulator OxyR. In the laboratory adapted H37RV strains, both of the OxyR and AhpC genes were divergently transcribed, but the OxyR gene was found to be inactivated by multiple lesions including frameshifts , deletions and stop codons that ablated any gene function.

This result was confirmed in all M. tuberculosis strains tested and in all members of the $M$. tuberculosis complex. As added proof, inactivation of the OxyR or AhpC in $E$. coli will also confer INH susceptibility to this otherwise insensitive bacteria (55). When considering the above evidence, it appears that $M$. tuberculosis relies heavily on the defense afforded by their novel cell wall, having essentially eliminated most of the oxidative stress response (except KatG) from their genome.
Naturally, one must consider that the major mutations associated with INH resistance also cause reduced catalase-peroxidase activity in $\mathrm{KatG}$, this, according to Sherman et al. is paradoxical since KatG is intimately involved in the survival and pathogenesis of $M$. tuberculosis (53). This group found a strong synergistic effect of $\mathrm{H} 2 \mathrm{O} 2$ and INH in KatG positive BCG strains. They conducted a series of expression studies and a preinduced KatG was capable of increasing survival 35 fold. To determine the compensatory mechanism in KatG (catalase negative) situations, Shermin examined 8 clinical $\mathrm{INH}^{\mathrm{R}}$ KatG mutants for altered expression profiles. All 8 isolates expressed a $22 \mathrm{KD}$ protein at considerably higher levels than H37RV controls. This protein was found to be AhpC. Its hyperexpression was a result of point mutations within its promoter region that apparently abrogate the requirement for OxyR regulatory control. The temporal appearance of this mutation was investigated and appears to be the result of a second in vivo selection event after KatG mutation. Several groups proposed that the increased AhpC expression could play multiple roles in vivo $(53,54)$. First, it could directly counteract INH effect, or simply compensate for loss of KatG activity thereby increasing overall viability. Sherman reported that while AhpC upregulation provides substantial benefit against peroxide insult it does not detoxify INH. Conversely, Zhang et al. supports an independent role for AhpC mutations in the emergence of low INH resistance (54).

The relevance of this mutation should also be questioned; particularly in light of two recent clinically based studies $(56,57)$. The first, sequenced the AxyRAhpC region of 229 M. tuberculosis isolates recovered from infected humans and animals, where the KatG and InhA regions had been sequenced and reported previously (56). The most important feature of this study was that most $\mathrm{INH}^{\mathrm{R}}$ strains carrying substantially reduced activity of KatG (ie. 315 mutation) lacked any alterations in AhpC or OxyR-AhpC intergenic regions. The second study analysed 57 clinical isolates. Here, 8 compensatory AhpC promoter mutations were identified in 8 catalase negative KatG defective strains whereas the corresponding region of 25 catalase positive $\mathrm{INH}^{\mathrm{R}}$ isolates were unaltered (57). Taken together, these results show little evidence for an independent role for 


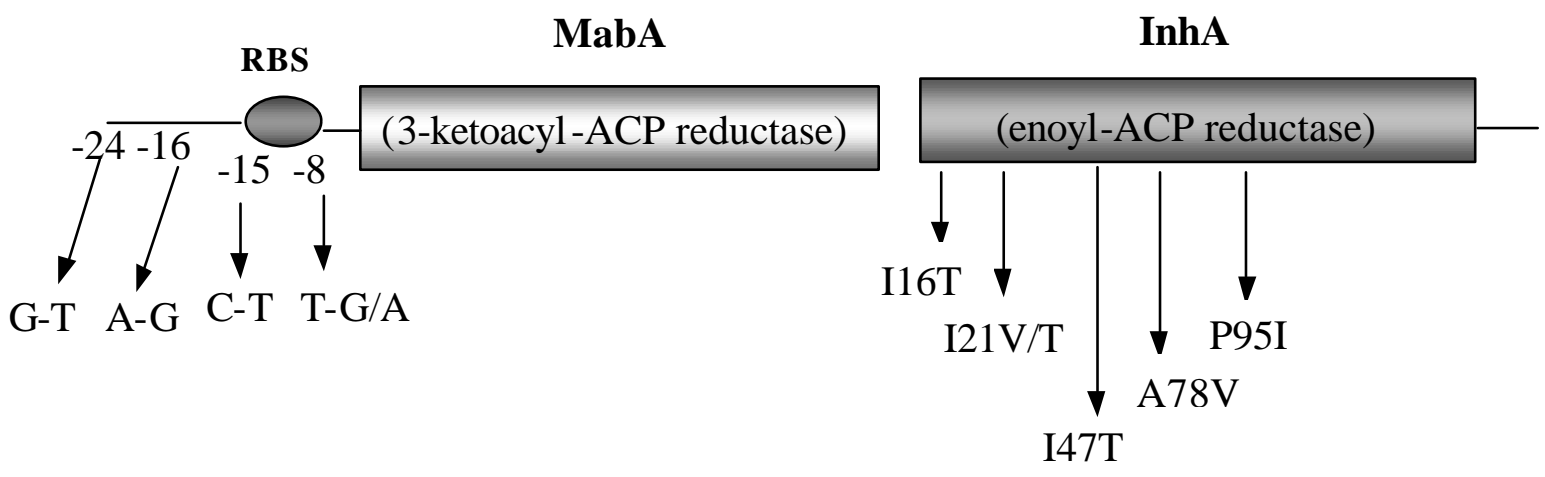

Figure 4. InhA and upstream regulatory element mutations implicated in INH resistance Adapted from S. Ramasaswamy et al 1998.

AhpC in INH resistance which appears at a low frequency similar to what is found in catalase negative strains. These strains may be of clinical significance.

\section{THE "GLOBAL" APPROACH TO ISONIAZID RESISTANCE}

Collectively, it is apparent there is controversy over the identification of the molecular correlates of resistance of MTB to isoniazid. There is also some lack of agreement concerning several mutations and/or polymorphisms being causally linked and clinically relevant to INH resistance. The main problem appears to be that previous genotypic studies of $M$. tuberculosis may have incorrectly estimated the importance of specific drug mutations due to errors in sampling, as well as the inclusion of MDR isolates and inappropriate controls. Also, the biochemical characterization of many proposed targets (resistant or susceptible) has inevitably used non-representative lab strains or nonpathogenic species for ease and safety, which may not accurately reflect that seen in the infected individual.

These errors, combined with a reductionist approach to drug resistance, has only revealed a fragmented and convoluted picture of $M$. tuberculosis resistance to isoniazid. To alleviate further discrepancies, a more holistic or "global" approach should be taken that makes full use of genomic databases, tools and methods. A comprehensive review of this subject can be obtained from several sources (58-65).

Several recent studies have undertaken this approach and have yielded a wealth of information. The first study by Wilson et al. uses micro-array hybridization to determine changes in expression patterns under INH exposure (66).

The basic methodology is summarized elsewhere, however in brief, at time zero (pre INH) and each successive time point (post INH), mRNA was isolated pelleted mycobacterial culture. These RNA species were differentially labeled with fluorochrome tags in a reverse transcriptase reaction (RT) to produce cDNAs that were used as probes for microarray hybridization.
The resulting pattern is analysed via software and intensity changes allow discrimination between relative changes in mRNA expression.

This enabled the documentation of a highly induced gene cluster encoding components of the FAS II fatty acid operon. Several of these genes (acpM and kasA) have been previously reported, corroborating previous studies, and the methods used in this study. The induction of these genes were observed as early as twenty minutes post- treatment. Three additional INH induced proteins were also reported, the first of which is $\mathrm{FbpC}$ which is an abundant exported protein involved in mycolic acid maturation in the outer wall. FbpC has also been previously reported $(67,68)$ since it constitutes one of the 3 highly homologous proteins comprising the $85 \mathrm{C} \mathrm{Ag}$ complex. As an added note, this complex has been proposed as the site of interaction with human fibronectin and therefore is a primary target for rational drug design and vaccines.

Several novel proteins lacking characterization were also found, 2 acetyl-CoA dehydrogenases (FADE23, FADE24) and an efflux protein EfpA as well as a subunit of AhpC. Further investigation of the relevance of transcriptional responses was analyzed in $\mathrm{INH}^{\mathrm{R}}$ strains under conditions of INH and ETH treatment. The first results of this experiment showed a similar upregulation pattern by ETH as observed by INH. The second experiment of $\mathrm{INH}^{\mathrm{R}}$ strains revealed no significant alteration of gene expression upon INH insult. Taken together, these results agree with previous literature yet provide a more complete view of all the players involved and the inclusion of several new therapeutic and prophylactic targets.

A similar approach was employed by Allard using a differential expression customized amplification library (Decal) (69). One important feature of this method is the ability to resolve four-fold differences in mRNA expression without confounding from constitutively expressed mRNA (housekeeping genes). Although beyond the scope of this paper, an additional method described by Brenner et al. allows the resolution of "a 
few tens of mRNA copies per cell" (70). Both methods are quite amenable to bacterial systems as they do not rely on poly adenylation of mRNA transcripts and both require no previous sequence knowledge.

The Alland paper was directed specifically towards the effect of isoniazid on the genomic expression of Mycobacterium tuberculosis, similar to the work of the Wilson group. Their results showed an upregulation of three previously unknown isoniazid-induced genes, IniA, IniB and IniC, all of which have putative functions in cell wall synthesis or a hypothesized protective role in response to cell wall destruction. A later report has characterized the rather unique ini BAC promoter. A molecular genetic analysis described a regulatory region and putative repressor that was specifically induced by a variety of cell wall inhibitors, but only in actively replicating cells $(71,74)$.

The final report by Piatek et al. is an excellent example of the use of a molecular epidemiological approach to drug resistance (72). The work utilized a PCR based molecular beacon assay. Briefly, this assay utilizes specifically paired fluorogenic PCR primers which allows real time monitoring of multiple PCR products in a single reaction (i.e. multiplex PCR), The assay itself is relatively quick and results can be analyzed within several hours $(72,73)$.

The populations used in this study were distinct. The first was from a reference lab in Spain. This population was known to have an overrepresentation of MDR clinical isolates. The second study population was from a community medical center in New York. The use of these two populations was to contrast and thereby preclude many previous mistakes in assessing the clinical relevance of mutations. The study was also used to characterize the assay and it was found to be particularly effective as a predictive screen for $\mathrm{INH}^{\mathrm{R}}$ with a sensitivity and specificity of $85 \%$ and $100 \%$ respectively for detection of mutations in the KatG, InhA and AhpC loci. Results of stratified analysis of the sample populations showed dramatic differences in the ability to discriminate $\mathrm{INH}^{\mathrm{R}}$. Ninety-four percent of isolates from Spain contained mutations associated with $\mathrm{INH}^{\mathrm{R}}$ versus only seventy-six percent of New York isolates. Analysis to exclude the confounding of MDR revealed $94 \%$ and $96 \%$ of isolated from Madrid and New York respectively contained INH resistance mutations. Restriction to New York isolates only showed a strong correlation of $\mathrm{INH}^{\mathrm{R}}$ and MDR (94\%) compared to only $44 \%$ of single drug resistance (i.e. $\mathrm{INH}^{\mathrm{R}}$ ) mutations.

The authors then investigated the possibility of the KasA mutation accounting for the discrepancies. No mutations were found in codons $(66,312,413)$ previously reported to be associated with INH $\mathrm{r}$ (48).
One previously reported mutation, G269S, was found in 10 isolates but were equally distributed between resistance and susceptible controls, suggesting no association with $\mathrm{INH}^{\mathrm{R}}$. As previously mentioned for $\mathrm{KatG}$, this likely constitutes a polymorphism.

The authors went on to address the possibility of $\mathrm{INH}^{\mathrm{R}}$ being the end result of a temporal sequence of mutations that have previously been unassociated with resistance. The hypothesis of a series cumulative mutations (i.e.."a genetic barrier") imparting resistance while interesting, is not a novel one, and in this particular situation it remains yet unfounded.

\section{FUTURE DIRECTIONS}

To resolve the previously stated issues, in the interests of public health will require accurate assessment of resistance mutations, polymorphisms, and their relevance (i.e. those being both sufficient and necessary to effect drug resistance) in a clinical setting. Although the primary target(s) of INH are elements of the FASII system, necessary for mycolic acid synthesis, the predominance of mutations seen in patients seem to be localized to KatG.

Further study of these and related problems using burgeoning new technologies, and information focusing on molecular pathogenesis should provide important new insights into mycobacterial metabolism, the genetic basis of mycobacterial drug resistance, and host response. This approach will inevitably lead to new targets, and drug leads. These will hopefully translate to prophylactic and therapeutic interventions in the next several years.

\section{ACKNOWLEDGMENTS}

We acknowledge Dr. M. Götte for helpful comments in this manuscript. This work was aided by a CIHR Doctoral Fellowship to J.B.W.

\section{REFERENCES}

1. Dye C, Scheele S, Dolin P, Pathania V, Raviglione MC. Consensus statement. Global burden of tuberculosis: estimated incidence, prevalence, and mortality by country. WHO Global Surveillance and Monitoring Project JAMA. 1999; 282(7): 677 86.

2. Dolin P.J., Raviglione M.C. and Kochi A., Global tuberculosis incedence and mortality during 1990-2000. Bull WHO 72: 213220.

3. Harrison's Principles of Internal Medicine 15th edition, vol1 pgs 1024-1033. 2000.

4. Small PM, Fujiwara PI. Management of tuberculosis in the United States. N Engl J Med; 2001 345(3): 189-200.

5. Mahmoudi A, Iseman MD. Pitfalls in the care of patients with tuberculosis. Common errors and their association with the acquisition of drug resistance. JAMA. 1993; 270(1): 65-8.

6. Moss AR, Hahn JA, Tulsky JP, Daley CL, Small PM, Hopewell 
PC. Tuberculosis in the homeless. A prospective study. Am J Respir Crit Care Med. 2000;162( 2 Pt 1): 460-4.

7. Prospects for vaccines to protect against AIDS, tuberculosis, and malaria. Letvin NL, Bloom BR, Hoffman SL. JAMA. 2001; 285(5): 606-11.

8. Tuberculosis and HIV infection in sub-Saharan Africa. De Cock KM, Soro B, Coulibaly IM, Lucas SB. JAMA. 1992; 268(12): 1581-71.

9. Cole ST, Brosch R, Parkhill J, Garnier T, Churcher C, Harris D, Gordon SV, Eiglmeier K, Gas S, Barry CE 3rd, Tekaia F, Badcock K, Basham D, Brown D, Chillingworth T, Connor R, Davies R, Devlin K, Feltwell T, Gentles S, Hamlin N, Holroyd S, Hornsby, Jagels K, Barrell BG. Deciphering the biology of Mycobacterium tuberculosis from the complete genome sequence Nature. 1998; 393(6685): 537-44.

10. Philipp WJ, Poulet S, Eiglmeier K, Pascopella L, Balasubramanian V, Heym B, Bergh S, Bloom BR, Jacobs WR Jr, Cole ST. An integrated map of the genome of the tubercle bacillus, Mycobacterium tuberculosis H37Rv, and comparison with Mycobacterium leprae Proc Natl Acad Sci U S A. 1996; 93(7): 3132-7.

11. Sterling TR, Brehm WT, Moore RD, Chaisson RE. Tuberculosis vaccination versus isoniazid preventive therapy: a decision analysis to determine the preferred strategy of tuberculosis prevention in HIVinfected adults in the developing world. Int J Tuberc Lung Dis. 1999; 3(3): 248-54

12. Nolan CM, Goldberg SV, Buskin SE. Hepatotoxicity associated with isoniazid preventive therapy: a 7-year survey from a public health tuberculosis clinic JAMA. 1999; 281(11): 1014-8.

13. Gurumurthy P, Ramachandran G, Vijayalakshmi S, Kumar AK, Venkatesan P, Chandrasekaran V, Vjayasekaran V Kumaraswami V, Prabhakar R. Bioavailability of rifampicin, isoniazid and pyrazinamide in a triple drug formulation: comparison of plasma and urine kinetics. Int J Tuberc Lung Dis. 1999; 3(2): 119-25.

14. Bass JB Jr, Farer LS, Hopewell PC, O'Brien R, Jacobs RF, Ruben F, Snider DE Jr, Thornton G. Treatment of tuberculosis and tuberculosis infection in adults and children. American Thoracic Society and The Centers for Disease Control and Prevention. Am J Respir Crit Care Med. 1994; 149(5):1359-74.

15. Bardou F, Raynaud C, Ramos C, Laneelle MA, Laneelle G. Mechanism of isoniazid uptake in Mycobacterium tuberculosis. Microbiology. 1998; 144 (9): 2539-44.

16. Musser JM. Antimicrobial agent resistance in mycobacteria: molecular genetic insights. Clin Microbiol Rev. 1995; 8(4): 496-514

17. Middlebrook G. Isoniazid-resistance and catalase activity of tubercle bacilli Am Rev Tuberc. 1954; 69:471-472

18. Loewen P.C. Klotz MG, Hassett DJ, an old enzyme that continues to surprise us. ASM News. 2000; 66:76-82.

19. Li Z, Kelley C, Collins F, Rouse D, Morris S. Expression of katG in Mycobacterium tuberculosis is associated with its growth and persistence in mice and guinea pigs. J Infect Dis. 1998; 177(4): 10305.

20. Shoeb HA, Bowman BU Jr, Ottolenghi AC, Merola AJ. Peroxidasemediated oxidation of isoniazid. Antimicrob Agents Chemother. 1985; 27(3): 399-403.

21 Shoeb HA, Bowman BU Jr, Ottolenghi AC, Merola AJ. Evidence for the generation of active oxygen by isoniazid treatment of extracts of Mycobacterium tuberculosis H37Ra. Antimicrob Agents Chemother. 1985; 27(3):404 -7.

22. Zhang Y, Heym B, Allen B, Young D, Cole S. The catalase-peroxidase gene and isoniazid resistance of Mycobacterium tuberculosis. Nature. 1992; 358(6387): 591-3.

23. Nikaido H. Prevention of drug access to bacterial targets: permeability barriers and active efflux. Science. 1994; 264(5157): 382-8.

24. Spratt BG. Resistance to antibiotics mediated by target alterations Science. 1994; 264(5157): 388-93.

25. Davies J. Inactivation of antibiotics and the dissemination of resistance genes. Science. 1994; 64(5157): 375-82.

26. Heym B, Alzari PM, Honore N, Cole ST Missense mutations in the catalase-peroxidase gene, $\mathrm{katG}$, are associate $\mathrm{d}$ with isoniazid resistance in Mycobacterium tuberculosis Mol Microbiol. 1995;15(2): 235-45.

27. Cockerill FR 3rd. Genetic methods for assessing antimicrobial resistance. Antimicrob Agents Chemother. 1999; 43 (2): 199-212.

28. Olive M.D., Bean P. Principles and applications of methods for DNAbased typing of microbial organisms. J Clin Micro. 1999; 37(6): 16611669.

29. Pretorius GS, van Helden PD, Sirgel F, Eisenach KD, Victor TC. Mutations in katG gene sequences in isoniazid-resistant clinical isolates of Mycobacterium tuberculosis are rare. Antimicrob Agents Chemother. 1995; 39(10): 2276-81.

30. Marttila HJ, Soini H, Eerola E, Vyshnevskaya E, Vyshnevskiy BI, Otten TF, Vasilyef AV, Viljanen MK. A Ser315Thr substitution in $\mathrm{KatG}$ is predominant in genetically heterogeneous multidrug-resistant Mycobacterium tuberculosis isolates originating from the St. Petersburg area in Russia. Antimicrob Agents Chemother. 1998; 42(9): 2443-5.

31. Marttila HJ, Soini H, Huovinen P, Viljanen MK. katG mutations in isoniazid-resistant Mycobacterium tuberculosis isolates recovered from Finnish patients. Antimicrob Agents Chemother. 1996; 40(9): 2187-9.

32. van Soolingen D, de Haas PE, van Doorn HR, Kuijper E, Rinder H, Borgdorff MW. Mutations at Amino Acid Position 315 of the katG Gene Are Associated with High-Level Resistance to Isoniazid, Other Drug Resistance, and Successful Transmission of Mycobacterium tuberculosis in The Netherlands. J Infect Dis 2000; 182(6):1788-1790.

33. Rouse DA, Li Z, Bai GH, Morris SL. Characterization of the katG and inhA genes of isoniazid-resistant clinical isolates of Mycobacterium tuberculosis. Antimicrob Agents Chemother. 1995; 39(11):2472-7.

34. Haas WH, Schilke K, Brand J, Amthor B, Weyer K, Fourie PB, Bretzel G, Sticht-Groh V, Bremer HJ. Molecular analysis of katG gene mutations in strains of Mycobacterium tuberculosis complex from Africa. Antimicrob Agents Chemother. 1997; 41(7): 1601-3.

35. Musser JM, Kapur V, Williams DL, Kreiswirth BN, van Soolingen D, van Embden JD. Characterization of the catalase-peroxidase gene (katG) and inhA locus in isoniazid-resistant and -susceptible strains of Mycobacterium tuberculosis by automated DNA sequencing: restricted array of mutations associated with drug resistance. J Infect Dis. 1996; 173(1):196-202.

36. Rouse DA, DeVito JA, Li Z, Byer H, Morris SL Site-directed mutagenesis of the katG gene of Mycobacterium tuberculosis: effects on catalase-peroxidase activities and isoniazid resistance. Mol Microbiol. 1996; 22(3): 583-92.

37. Behr MA, Wilson MA, Gill WP, Salamon H, Schoolnik GK, Rane S, Small PM.. Comparative genomics of BCG vaccines by wholegenome DNA microarray. Science. 1999; 284(5419): 1520-3.

38. Behr MA, Small PM. A historical and molecular phylogeny of BCG strains. Vaccine; 1999; 17(7-8): 915-22.

39. Wengenack NL, Todorovic S, Yu L, Rusnak F. Evidence for differential binding of isoniazid by Mycobacterium tuberculosis KatG and the isoniazid-resistant mutant KatG (S315T). Biochemistry. 1998; 37(45): 15825-34.

40. Johnsson K, Froland WA, Schultz PG. Overexpression, purification, and characterization of the catalase-peroxidase KatG from Mycobacterium tuberculosis J Biol Chem. 1997; 272(5): 2834-40.

41. Van Doorn HR, Kuijper EJ, van Der Ende A, Welten AG, van Soolingen D, de Haas PE, Dankert J The Susceptibility of Mycobacterium tuberculosis to Isoniazid and the Arg-->Leu Mutation at Codon 463 of katG Are Not Associated. J Clin Microbiol. 2001; 39(4): 1591-4

42. Banerjee A, Dubnau E, Quemard A, Balasubramanian V, Um KS, Wilson T, Collins D, de Lisle G, Jacobs WR Jr. inhA, a gene encoding a target for isoniazid and ethionamide in Mycobacterium tuberculosis. Science. 1994; 263(5144): 227-30.

43 Dessen A, Quemard A, Blanchard JS, Jacobs WR Jr, Sacchettini JC. Crystal structure and function of the isoniazid target of Mycobacterium tuberculosis. Science. 1995; 267(5204): 1638-41. 
44. Rozwarski DA, Grant GA, Barton DHR, Jacobs WR Jr, Sacchettini JC. Modification of the NADH of the isoniazid target (InhA) from Mycobacterium tuberculosis. Science. 1998; 279(5347): 98-102.

45. Lei B, Wei CJ, Tu SC. Action mechanism of antitubercular isoniazid. Activation by Mycobacterium tuberculosis $\mathrm{KatG}$, isolation, and characterization of inhA inhibitor J Biol Chem. 2000; 275(4): 2520-6.

46. Mdluli K, Sherman DR, Hickey MJ, Kreiswirth BN, Morris S, Stover $\mathrm{CK}$, Barry CE 3rd. Biochemical and genetic data suggest that InhA is not the primary target for activated isoniazid in Mycobacterium tuberculosis. J Infect Dis. 1996; 174(5): 1085-90.

47. Mdluli K, Slayden RA, Zhu Y, Ramaswamy S, Pan X, Mead D, Crane DD, Musser JM, Barry CE 3rd. Inhibition of a Mycobacterium tuberculosis beta-ketoacyl ACP synthase by isoniazid. Science. 1998; 280(5369): 1607-10.

48. Lee AS, Lim IH, Tang LL, Telenti A, Wong SY. Contribution of kasA analysis to detection of isoniazid-resistant Mycobacterium tuberculosis in Singapore. Antimicrob Agents Chemother. 1999; 43(8): 2087-9.

49. Slayden RA, Lee RE, Barry CE 3rd. Isoniazid affects multiple components of the type II fatty acid synthase system of Mycobacterium tuberculosis. Mol Microbiol. 2000; 38(3): 514-25.

50. Sherman DR, Sabo PJ, Hickey MJ, Arain TM, Mahairas GG, Yuan Y, Barry CE 3rd, Stover CK. Disparate responses to oxidative stress in saprophytic and pathogenic mycobacteria. Proc Natl Aced Sci U S A . 1995; 92(14): 6625-9.

51. Deretic V, Philipp W, Dhandayuthapani S, Mudd MH, Curcic R, Garbe T, Heym B, Via LE, Cole ST. Mycobacterium tuberculosis is a natural mutant with an inactivated oxidative-stress regulatory gene: implications for sensitivity to isoniazid. Mol. Microbiol. 1995; 17(5): 889-900.

52. Dhandayuthapani S, Mudd M, Deretic V. Interactions of OxyR with the promoter region of the oxyR and ahpC genes from Mycobacterium leprae and Mycobacterium tuberculosis. J Bacteriol. 1997; 179(7):2401-9.

53. Sherman DR, Mdluli K, Hickey MJ, Arain TM, Morris SL, Barry CE 3rd, Stover CK. Compensatory ahpC gene expression in isoniazidresistant Mycobacterium tuberculosis. Science. 1996; 272(5268): 1641-3.

54. Zhang Y, Dhandayuthapani S, Deretic V. Molecular basis for the exquisite sensitivity of Mycobacterium tuberculosis to isoniazid Proc Natl Acad Sci U S A. 1996; 93(23): 13212-6.

55. Rosner JL, Storz G. Effects of peroxides on susceptibilities of Escherichia coli and Mycobacterium smegmatis to isoniazid. Antimicrob Agents Chemother. 1994; 38(8): 1829-33.

56. Sreevatsan S, Pan X, Zhang Y, Deretic V, Musser JM. Analysis of the oxyR-ahpC region in isoniazid-resistant and -susceptible Mycobacterium tuberculosis complex organisms recovered from diseased humans and animals in diverse localities Antimicrob Agents Chemother. 1997; 41(3): 600-6.

57. Kelley CL, Rouse DA, Morris SL. Analysis of ahpC gene mutations in isoniazid-resistant clinical isolates of Mycobacterium tuberculosis Antimicrob Agents Chemother. 1997; 41(9): 2057-8.

58. Duggan DJ, Bittner M, Chen Y, Meltzer P, Trent JM Expression profiling using cDNA microarrays. Nat Genet. 1999; 21(1 Suppl): 104.

59. Arigoni F, Talabot F, Peitsch M, Edgerton MD, Meldrum E, Allet E, Fish R, Jamotte T, Curchod ML, Loferer H A genome-based approach for the identification of essential bacterial genes. Nat Biotechnol. 1998; 16(9): 851-6.

60. Lennon GG. High-throughput gene expression analysis for drug discovery Drug Discov Today. 2000; 5(2): 59-66.

61. Moir DT, Shaw KJ, Hare RS, Vovis GF. Genomics and antimicrobial drug discovery. Antimicrob Agents Chemother. 1999; 43(3): 439-46.

62. Debouck C, Goodfellow PN. DNA microarrays in drug discovery and development Nat Genet. 1999; 21(1 Suppl):48-50.

63. Rosamond J, Allsop A. Harnessing the power of the genome in the search for new antibiotics. Science. 2000; 287(5460): 1973-6.

64. Lipshutz RJ, Fodor SP, Gingeras TR, Lockhart DJ. High density synthetic oligonucleotide arrays. Nat Genet. 1999; 21(1 Suppl): 20-4.

65. Brent R. Genomic biology Cell. 2000; 100(1): 169-83.

66. Wilson M, DeRisi J, Kristensen HH, Imboden P, Rane S, Brown PO, Schoolnik GK. Exploring drug-induced alterations in gene expression in Mycobacterium tuberculosis by microarray hybridization Proc Natl Acad Sci U S A. 1999; 96(22): 12833-8.

67. Garbe TR, Hibler NS, Deretic V. Isoniazid induces expression of the antigen 85 complex in Mycobacterium tuberculosis Antimicrob Agents Chemother. 1996; 40(7):1754-6.

68. Ronning DR, Klabunde T, Besra GS, Vissa VD, Belisle JT, Sacchettini JC. Crystal structure of the secreted form of antigen $85 \mathrm{C}$ reveals potential targets for mycobacterial drugs and vaccines. Nat Struct Biol. 2000; 7(2): 141-6.

69. Alland D, Kramnik I, Weisbrod TR, Otsubo L, Cerny R, Miller LP, Jacobs WR Jr, Bloom BR. Identification of differentially expressed mRNA in prokaryotic organisms by customized amplification libraries (DECAL): the effect of isoniazid on gene expression in Mycobacterium tuberculosis. Proc Natl Acad Sci U S A. 1998; 95(22): 13227-32.

70. Brenner S, Williams SR, Vermaas EH, Storck T, Moon K, McCollum C, Mao JI, Luo S, Kirchner JJ, Eletr S, DuBridge RB, Burcham T, Albrecht G. In vitro cloning of complex mixtures of DNA on microbeads: physical separation of differentially expressed cDNAs Proc Natl Acad Sci U S A. 2000; 97(4): 1665-70.

71. Alland D, Steyn AJ, Weisbrod T, Aldrich K, Jacobs WR Jr. Characterization of the Mycobacterium tuberculosis iniBAC promoter, a promoter that responds to cell wall biosynthesis inhibition. J Bacteriol. 2000; 182(7): 1802-11.

72. Piatek AS, Telenti A, Murray MR, El-Hajj H, Jacobs WR Jr, Kramer FR, Alland D.Genotypic analysis of Mycobacterium tuberculosis in two distinct populations using molecular beacons: implications for rapid susceptibility testing Antimicrob Agents Chemother. 2000; 44(1):103-10.

73. Tyagi S, Marras SA, Kramer FR. Wavelength-shifting molecular beacons. Nat Biotechnol. 2000; 18(11): 1191-6.

74. Slayden RA, Barry CE 3rd The genetics and biochemistry of isoniazid resisitance in Mycobacterium tuberculosis. Microbes Infect 2000; 2(6): 659-69

James B. Whitney received his BSc. Degree in Microbiology from the University of Manitoba. He is currently engaged in the third year of a Ph.D. in Microbiology and Immunology at the McGill AIDS Centre, (Montreal Quebec). His research focuses on live-attenuated vaccine models for HIV. Dr. Mark A. Wainberg is the director of the McGill AIDS Centre, he is also the scientific director of the Lady Davis Institute for Medical Research in Montreal. 